\title{
Lokale Agenda 21 - partizipative Planung nachhaltiger Stadtentwicklung?
}

\section{Zur Karriere eines Begriffs}

Der Begriff «Nachhaltigkeit» hat in den letzten Jahren die bereits etwas abgenutzten Begriffe «Umwelt» und «Lebensqualität» als modische Schlagworte in der Planungsdiskussion verdrängt. Wenn man Schlagworte als charakteristische Ausdrucksform des Zeitgeistes begreift, der durch sie das intellektuelle Bewußtsein möglichst vieler Zeitgenossen schlagen und treffen will (HUBER 1976, 15), dann ist zunächst zu fragen, wie dieser Zeitgeist entstanden ist.

Vor dem Hintergrund sich abzeichnender globaler Verteilungskämpfe (z. B. Nord-Süd-Konflikt) und Umweltkatastrophen (z.B. Klimaerwärmung) haben die UN eine Weltkommission für Umwelt und Entwicklung eingesetzt. Im Schlußbericht von 1987 - nach der Vorsitzenden der Kommission Brundtland-Report genannt wurde der Begriff «Sustainable Development» verwendet. Darunter wird eine Entwicklung verstanden, welche die Bedürfnisse der Gegenwart befriedigt, ohne aber $\mathrm{zu}$ riskieren, daß künftige Generationen ihre eigenen Bedürfnisse nicht mehr befriedigen können (NIEMANN 1997, 31). In deutscher Übersetzung wird dafür meist der Begriff «nachhaltige Entwicklung» (aber auch «zukunftsfähige» oder «zukunftsbeständige» Entwicklung) verwendet.

1992 wurden auf dem «Erdgipfel» der UN (Konferenz zu Umwelt und Entwicklung) Grundlagen einer qualitativ neuen, weltweiten Zusammenarbeit diskutiert. Die Ergebnisse wurden in einem Aktionsprogramm für das 21 . Jahrhundert zusammengefaßt und von über 170 Staaten verabschiedet. Diese Agenda 21 umfaßt in 40 Kapiteln alle bekannten Politikbereiche einer umweltverträglichen Entwicklung. In der Übersetzung des deutschen Umweltministeriums (Bundesministerium für Umwelt, Naturschutz und Reaktorsicherheit, o. J.) umfaßt die Agenda 21 fast 300 Seiten.

\section{Von der Agenda 21 zur Lokalen Agenda 21}

Im Kapitel 28 der Agenda 21: «Initiativen der Kommunen zur Unterstützung der Agenda 21» (Bundesministerium für Umwelt, Naturschutz und Reaktorsicherheit, o. J., 231) wird festgehalten, daß die Umsetzung auf kommunaler Ebene erfolgen müsse, «da viele der in der Agenda 21 angesprochenen Probleme und Lösungen auf Aktivitäten auf der örtlichen Ebene zurückzuführen sind» (§28.1). Deshalb soll «jede Kommunal- verwaltung (...) in einen Dialog mit ihren Bürgern, örtlichen Organisationen und der Privatwirtschaft eintreten und eine 〈kommunale Agenda 21 > beschließen» $(\S 28.3)$. NIEMANN $(1997,32)$ weist in diesem Zusammenhang darauf hin, daß das englische «Local Agenda 21» zunächst als lokal statt kommunal übersetzt wurde, so daß sich die - eigentlich falsche - Bezeichnung «Lokale Agenda 21» einprägte.

Gemäß §28.2, Abs. a sollte sich bis 1996 «die Mehrzahl der Kommunalverwaltungen der einzelnen Länder gemeinsam mit ihren Bürgern einem Konsultationsproze $\beta$ unterzogen haben und einen Konsens hinsichtlich einer «kommunalen Agenda 21〉 für die Gemeinschaft erzielt haben» (BUNDESMINISTERIUM FÜR UMWELT, NATURSCHUTZ UND REAKTORSICHERHEIT, O. J., 231). Dieses Ziel wurde bis heute nicht erreicht. Zwar befaßten sich weltweit tätige Organisationen, wie der International Council for Local Environmental Initiatives (ICLEI), mit der von den UN gewünschten Ausformulierung des Kapitels 28 der Agenda 21. Diese Charta von Aalborg wurde 1994 von der Konferenz europäischer Städte verabschiedet und von über 200 Städten unterzeichnet (NIEMANN 1997, 33).

Die komplexe Agenda 21 auf kommunaler Ebene umzusetzen ist allerdings äußerst schwierig. Dies mag bisher viele Kommunen davon abgehalten haben, sich auf ein solches «Wagnis» einzulassen. Zwar stellte ICLEI (1995) den Unterzeichnern der Charta von Aalborg einen Leitfaden zur Erarbeitung einer LA 21 zur Verfügung. Dieser befaßte sich aber fast ausschließlich mit Umweltaspekten. Nun sollte eine LA 21 aber gleichgewichtig ökologische, ökonomische und soziale Aspekte berücksichtigen. Deshalb wurde in Deutschland versucht, konsensfähige Handlungsfelder abzustecken (vgl. z.B. DEUTSCHER STÄDTETAG 1995), die alle drei Aspekte berücksichtigen.

\section{Umsetzungsprobleme auf der kommunalen Ebene}

Möglichkeiten und Grenzen von Agenda-Prozessen wurden im Juni 1998 auf einer Tagung diskutiert, welche die Deutsche Vereinigung für Angewandte Geographie (DVAG) zum Thema «Alles Agenda oder was?» in

Lienhard Lötscher, Prof. Dr., Ruhr-Universität Bochum, Geographisches Institut, D-44780 Bochum

Kai Kühmichel, Dipl.-Ing., Architekt und Stadtplaner, Maelostraße 3, D-45894 Gelsenkirchen 


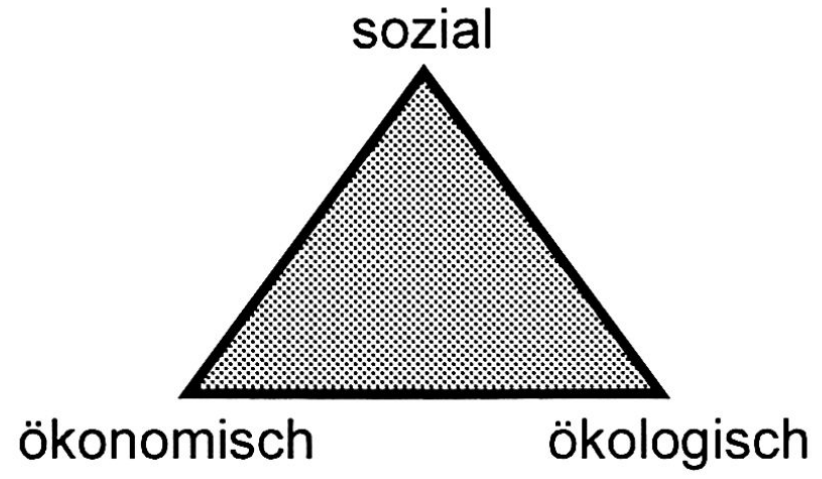

Abb. 1 Aspekte der Nachhaltigkeit.

Münster durchführte. In einer von L. Lötscher geleiteten Podiumsdiskussion wiesen die in verschiedenen Projekten engagierten Planer auf folgende Schwierigkeiten hin:

- zunächst sei es wegen der oft divergierenden Interessenlage der Beteiligten schwierig, aus einem «Sammelsurium» von Ideen ein inhaltliches Konzept zu erstellen, das als konsensfähige Leitlinie des Planungsprozesses Bestand habe

- solche Konzepte seien deshalb oft sehr umfangreich, so daß deren Erarbeitung sich über lange Zeiträume erstrecke (oft mehrere Jahre)

- eine weitere Schwierigkeit von Zukunftsplanungen sei, daß aktuelle Probleme oft ausgeklammert würden, wenn dazu keine konsensfähigen Lösungsansätze vorlägen

- wenn allerdings der «spontane Leidensdruck» gering sei, dann fehle bei vielen Bürgern die Motivation zur Mitarbeit

- deshalb sei es wichtig, daß konkrete Projekte angesprochen werden, die in absehbarer Zeit realisiert werden könnten, da sonst viele Bürger rasch das Interesse an den Planungsprozessen verlören

- die Art bzw. Form der Bürgerbeteiligung sei zudem oft entscheidend für Erfolg oder Mißerfolg eines Projektes

- so wurden zu den von ICLEI vorgeschlagenen Bürgerforen oft nur im voraus bekannte Interessenvertreter eingeladen (von deren Kompetenz einige der anwesenden Planungsexperten wenig überzeugt waren)

- für die Umsetzung der Konzepte sei aber eine möglichst breite Beteiligung wichtig, insbesondere von bereits existierenden Bürgerinitiativen und von Gruppierungen des dritten bzw. intermediären Sektors.

Die DVAG-Tagung in Münster zeigte, daß nach den bisherigen Erfahrungen ein Vorgehen am erfolgversprechendsten scheint, wenn es als von außen moderiertes Stadtentwicklungsprojekt angelegt wird, in dem im Sinne des Verfahrens einer Lokalen Agenda 21 ein möglichst breites Spektrum der Bürger mit Vertretern von Stadtverwaltung und Politikern zusammenarbeitet. Ein solches Projekt wird im folgenden vorgestellt.

\section{Zukunft Lüdinghausen - ein Beispiel partizipativer Stadtentwicklungsplanung}

Lüdinghausen, $30 \mathrm{~km}$ nördlich von Dortmund im südlichen Münsterland gelegen, ist eine Stadt von rund 23000 Einwohnern. Die Stadt will gut vorbereitet ins 21. Jahrhundert aufbrechen. Um in den Bereichen Stadtentwicklung und Stadtplanung zu klären, wie die derzeitige Funktion als traditionsreiches Mittelzentrum langfristig zu erhalten und auszubauen ist, forderten die Verantwortlichen ausgewählte Planungsbüros und Universitätsinstitute auf, Projektskizzen für die Erstellung eines neuen Stadtentwicklungskonzeptes einzureichen.

Ende August 1997 hat die Stadt die Projektskizze «Zukunft Lüdinghausen - Stadtprofil 2010» zur weiteren Bearbeitung ausgewählt. Sie ist vom Lehrstuhl für Kultur- und Siedlungsgeographie (L. Lötscher) der RuhrUniversität Bochum in Zusammenarbeit mit einem Architektur- und Planungsbüro (K. Kühmichel) und einem Büro für Unternehmensberatung (K. Kost) eingereicht worden. Die zweijährige Studie ist an aktuellen Entwicklungen der Planung, wie innovativer Bürgerbeteiligung und nachhaltiger Stadtplanung im Sinne einer Lokalen Agenda 21, orientiert. Ziel ist, den politischen Entscheidungsträgern wie auch der Stadtverwaltung ein - zusammen mit interessierten Bürgern - erarbeitetes Konzept in die Hand zu geben, das erlaubt, im Laufe der nächsten 10 Jahre Entscheidungen vor dem Hintergrund eines breiten planerischen Kontextes zu treffen, um eine konsequente, zukunftsfähige Entwicklung Lüdinghausens zu erreichen.

Die Erarbeitung des «Stadtprofils 2010» erfolgt in sechs Phasen:

1. Analysephase: entwicklungsrelevante Informationen sind zu ermitteln und bereits vorhandene planungsrelevante Konzepte und Unterlagen aufzuarbeiten.

2. Kommunikationsphase I: in Workshops werden innovative Beteiligungsformen angewendet, um zu erfahren, in welchen Themenfeldern Bürgerschaft, Wirtschaft, Politik und Verwaltung den dringendsten Handlungsbedarf zur Erreichung einer nachhaltigen Entwicklung sehen.

3. Kommunikationsphase II: in Zukunftswerkstätten werden innovative Beteiligungsformen angewendet, um herauszufinden, welche Entwicklungsziele Bürgerschaft, Wirtschaft, Politik und Verwaltung in welchen Themenfeldern anstreben.

4. Konzeptionsphase I: Informationen aus den Phasen 1 bis 3 werden in Szenarien gebündelt, um mögliche künftige Entwicklungen zu verdeutlichen.

5. Kommunikationsphase III: durch innovative Beteiligungsformen von Bürgerschaft, Wirtschaft, Politik und Verwaltung werden die konsensfähigen Inhalte der Szenarien ermittelt und die strittigen Punkte gegeneinander abgewogen. Zudem wird die Einführung eines künftigen «Monitoring Systems» diskutiert, das die Einhaltung der vereinbarten Entwicklungsziele für alle Bewohner transparent macht. 
6. Konzeptionsphase II: das «Stadtprofil 2010» wird in Form eines Berichtes erarbeitet und in einer Bürgerversammlung vorgestellt.

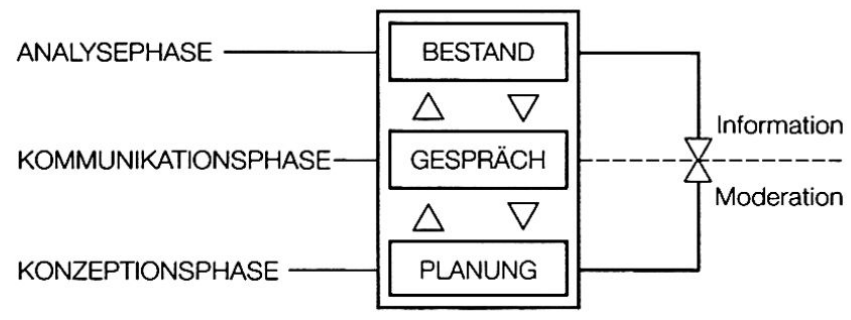

Abb. 2 Das Arbeitskonzept für das "Stadtprofil 2010».

Dieses prozessuale und partizipative Vorgehen läßt sich mit bisherigen Planungsverfahren kaum vergleichen. Der mehrstufige Beteiligungsprozeß unterscheidet sich auch von bisherigen Vorgehensweisen bei der Erarbeitung von LA 21 in deutschen Städten. Deshalb wird nachstehend die Methodik der einzelnen Arbeitsschritte zusammenfassend vorgestellt:

In den Workshops der Kommunikationsphase I wurde die Metaplantechnik eingesetzt, die sich als Instrument zur Steuerung von zielorientierten Gruppengesprächen bewährt hat. Diese Technik baut auf der Wahrnehmungserkenntnis auf, daß Visualisierung den Erfolg einer Vermittlungsarbeit wesentlich beeinflußt. Notizkarten und Pinnwände sind deshalb wichtige Hilfsmittel zur Problembenennung, Ideensammlung und Lösungsfindung.

Auf jedem Workshop wurde im Anschluß an eine «Aufwärmphase» den Teilnehmern die Frage gestellt: «Welche wichtigen Aufgaben wird die Stadt bis zum Jahre 2010 zu bewältigen haben?» In einem nachfolgenden individuellen (und stillen) Brainstorming war jeder Teilnehmer aufgefordert, spontan die drei wichtigsten Aufgaben auf je eine Notizkarte zu schreiben. Anschließend wurden die Karten eingesammelt und (anonym) entsprechend den Zurufen der Teilnehmer auf Pinnwänden angeordnet. Im nächsten Schritt wurden die so entstandenen Cluster nochmals entsprechend der Zurufe geordnet und deren Inhalte mit einer konsensfähigen Überschrift benannt. In einem weiteren Arbeitsschritt wurden die Teilnehmer aufgefordert, die so entstandenen Themenfelder durch die Vergabe von drei Klebepunkten (pro Teilnehmer) zu bewerten. Die auf diese Weise entstandene Rangfolge wurde mit der Gruppe diskutiert. Darauf konnten sich die Teilnehmer ein Arbeitsfeld aussuchen, das sie vertiefen wollten. So wurden zwei bis vier Kleingruppen gebildet. Die Diskussionsergebnisse wurden von Teilnehmern der Gesamtgruppe vorgestellt und danach besprochen. Nach einem kurzen Fazit der Moderatoren wurde das weitere Vorgehen entsprechend den oben genannten sechs Arbeitsphasen erläutert und mitgeteilt, wann das Gesamtergebnis der sechs Workshops in einer Bürgerversammlung vor- und zur Diskussion gestellt werde.
In diesen sechs Workshops wurden von den Teilnehmern insgesamt sieben Handlungsfelder ermittelt, in denen sie die wichtigsten Aufgaben der Stadt sahen, die im Laufe der nächsten 10 Jahre gelöst werden müßten. Nachstehend sind die Handlungsfelder nach der Gewichtung der Teilnehmer rangiert:

1. Wirtschaft und Arbeit

2. Planen, Bauen, Wohnen

3. Verkehr

4. Schule, Bildung und Soziales

5. Freizeit, Sport, Kultur

6. Umwelt und Ökologie

7. Tourismus.

Vor der Bürgerversammlung wurden alle Workshopteilnehmer persönlich angeschrieben. Sie wurde zudem in der Lokalpresse angekündigt. Erfreulicherweise war dann auch der Kapitelsaal der Burg Lüdinghausen bis auf den letzten Platz besetzt. Nach Meinung der Anwesenden waren die in den Workshops ermittelten Handlungsfelder zutreffend - und zumindest spontan nicht zu ergänzen. Bei der Gewichtung - jeder Anwesende konnte ein Handlungsfeld als erste, zweite oder dritte Priorität einstufen - räumte auch die Bürgerversammlung dem Handlungsfeld «Wirtschaft und Arbeit» die bei weitem höchste Priorität ein. An zweite Stelle rückte allerdings der Bereich «Verkehr» - mit einigem Abstand zu den übrigen Handlungsfeldern. Einen direkten Anhaltspunkt zu Problembereichen, die auf den oben erwähnten persönlichen Leidensdruck schließen ließen, erhielten wir dadurch, daß die Anwesenden gebeten wurden, zwei «Brennpunkte», die ihnen besonders am Herzen lagen, auf einer Übersichtskarte mit roten Klebepunkten einzutragen. Das so entstandene Verteilungsmuster wurde danach ausführlich besprochen. Anschließend wurde das weitere Vorgehen und insbesondere die Arbeitsweise in Zukunftswerkstätten erläutert. Da sich für alle Themenfelder ausreichend Interessenten fanden, boten wir für jeden Bereich eine Zukunftswerkstatt an. Zusätzlich wurde aus dem Kreis der Bürgerversammlung je eine Zukunftswerkstatt für das im Laufe der Gebietsreform (bis 1975) eingemeindete «Rosendorf» Seppenrade und zum Thema «Planung aus der Sicht von Frauen und Kindern» gewünscht.

Insgesamt wurden somit neun verschiedene Zukunftswerkstätten durchgeführt. Auf Wunsch der Teilnehmer waren dies Abendveranstaltungen von jeweils drei bis vier Stunden Dauer, wobei sich meist mehrere Folgeveranstaltungen anschlossen. In den Zukunftswerkstätten entwarfen Bürgerinnen und Bürger zusammen mit Vertretern aus Politik (Ratsmitgliedern) und Verwaltung wünschbare und zunächst auch utopisch erscheinende Zukünfte, um schließlich deren Umsetzungsmöglichkeiten zu prüfen. Sie wurden somit als «Fachleute in eigener Sache» tätig. In dieser dritten Phase der Erarbeitung des «Stadtprofils 2010» (siehe oben) galt es ja herauszufinden, welche Entwicklungsziele Bürgerschaft, Wirtschaft, Politik und Verwaltung in welchen Themenfeldern anstreben, um diese in die später zu entwickelnden Szenarien einfließen zu lassen. 
Nachhaltige Stadtentwicklung sollte nicht nur Erwachsene, sondern insbesondere auch Heranwachsende interessieren. Einerseits steht hier auch ihre Zukunft zur Debatte. Andererseits sind sie schon heute an die Aufgaben von morgen heranzuführen. Kinder und Jugendliche sind deshalb entsprechend ihren altersspezifischen Fähigkeiten am Planungsprozeß zu beteiligen. In $\mathrm{Zu}$ sammenarbeit mit Lehrern verschiedener Schulstufen ist das Interesse für die zukunftsfähige Entwicklung der eigenen Stadt insofern geweckt worden, als in verschiedenen Unterrichtsfächern Lehreinheiten und Veranstaltungen eingeplant und durchgeführt wurden, die das Problembewußtsein der Schüler für ihren unmittelbaren Lebensalltag schärfen sowie gemeinschaftsförderndes Denken und Handeln stärken sollten.

Bis im Januar 1999 wird aufgrund der Ergebnisse der Arbeitsphasen 1 bis 3 ein Szenario für eine nachhaltige Stadtentwicklung erstellt. Wirkungsketten, die verschiedene Entwicklungsvarianten in den einzelnen Handlungsfeldern auslösen könnten, werden mit Bürgern, Verwaltung und Politik ausführlich erörtert, wobei gemeinsam konsensfähige Perspektiven zu erarbeiten sind. Mit diesen Ergebnissen wird bis Juni 1999 ein sowohl ökologisch wie ökonomisch und sozial ausgewogenes (und deshalb konsensfähiges) Stadtentwicklungskonzept «Zukunft Lüdinghausen - Stadtprofil 2010» ausgearbeitet, das ingesamt im Sinne der partizipativen Planung einer Lokalen Agenda 21 erstellt wird. Um dessen Umsetzung als Leitlinie der Stadtentwicklung zu gewährleisten, ist zusammen mit den oben genannten Beteiligten ein «Monitoring System» einzurichten, damit Entscheidungen für alle Bewohner transparent gestaltet sowie wirkungsgerecht nachgehalten werden können.

\section{Fazit}

Was ist nun neu an dieser partizipativen Stadtentwicklungsplanung, wie sie am Beispiel der Stadt Lüdinghausen vorgestellt wurde?

Im Sinne einer Lokalen Agenda 21 ist im Vergleich zu bisherigen Verfahren neu:

- die ökologischen, ökonomischen und sozialen Aspekte sind gleichgewichtig zu behandeln

- es ist ein konsensfähiges Konzept zu erarbeiten

- die Bürgerbeteiligung erfolgt von Anfang an.

Im Vergleich zu bisherigen Verfahren der Erstellung einer Lokalen Agenda ist neu:

- von Anfang an erfolgt nicht nur eine Beteiligung von interessierten Bürgern, sondern eine Zusammenarbeit von Bürgern mit Vertretern aus Verwaltung und Lokalpolitik

- das Beteiligungsverfahren ist mehrstufig, um Rückkopplungen in mehreren Arbeitsschritten zu ermöglichen

- ein «Monitoring System», das die Einhaltung der vereinbarten Entwicklungsziele für alle Bewohner transparent macht, gewährleistet die Umsetzung des Stadt- entwicklungskonzeptes und gegebenenfalls dessen Modifizierung und Fortschreibung.

\section{Literatur}

BISCHOFF, A., SELLE, K. \& SINNING, H. (1995): Informieren, Beteiligen, Kooperieren: Kommunikation in Planungsprozessen. Eine Übersicht zu Formen, Verfahren, Methoden und Techniken. Kommunikation im Planungsprozeß, Bd. 1. Dortmund.

BUNDESFORSCHUNGSANSTALT FÜR LANDESKUNDE UND RAUMORDNUNG (1996): Städtebaulicher Bericht. Nachhaltige Stadtentwicklung - Herausforderungen an einen ressourcenschonenden und umweltverträglichen Städtebau. Bonn.

BUNDESMINISTERIUM FÜR RAUMORDNUNG; BAUWESEN UND STÄDTEBAU (Hrsg.) (1996): Lokale Agenda 21. Schriftenreihe "Forschung", des Bundesministeriums für Raumordnung, Bauwesen und Städtebau, Heft-Nr. 499. Bonn.

BUNDESMINISTERIUM FÜR RAUMORDNUNG; BAUWESEN UND STÄDTEBAU (Hrsg.) (1993): Zukunft Stadt 2000 Abschlußbericht. Bericht der Kommission Zukunft Stadt 2000. Bonn.

BUNDESMINISTERIUM FÜR RAUMORDNUNG; BAUWESEN UND STÄDTEBAU \& EMPIRICA (Hrsg.) (1998): Die Zukunft der Stadtregionen. Dokumentation eines Kongresses in Hannover am 22. und 23. Oktober 1997. Bonn.

BUNDESMINISTERIUM FÜR UMWELT, NATURSCHUTZ UND REAKTORSICHERHEIT (Hrsg.) (O.J.): Konferenz der Vereinten Nationen für Umwelt und Entwicklung im Juni 1992 in Rio de Janeiro - Dokumente - Agenda 21. Bonn.

DEUTSCHER STÄDTETAG (Hrsg.) (1995): Städte für eine umweltgerechte Entwicklung, Materialien für eine "Lokale Agenda 21". DST-Beiträge zur Stadtentwicklung und zum Umweltschutz, Reihe E, H. 24. Köln.

ELLWEIN, H. (1997): Bürgerbeteiligung und Partizipation heute und morgen: von der Bürgeranhörung zum Selbermachen. Informationen und Berichte zur Stadtentwicklung, Bd. 91. Essen.

HUBER, G. (1976): «Lebensqualität»: Modisches Schlagwort oder epochale Wende? In: Bättig, K., und Ermertz, E. (Hrsg.): Lebensqualität. poly 3. Basel: Birkhäuser, S. 15-26.

ICLEl (Hrsg.) (1995): European Local Agenda 21 Planning Guide - how to engage in long-term environmental action planning towards sustainability. Freiburg.

KALDUN, S. (1998): Von Rio de Janeiro nach Lüdinghausen (Teil 1): Fallbeispiel zur Lokalen Agenda 21. In: Die Wohnungswirtschaft, 51. Jg., H. 8/98, S. 23-24.

MINISTERIUM FÜR UMWELT, RAUMORDNUNG UND LANDWIRTSCHAFT DES LANDES NORDRHEIN-WESTFALEN (Hrsg.) (O.J.): Dokumentation des gegenwärtigen Diskussionsstandes und Handlungsbedarfs zum Thema Nachhaltigkeit und Zukunftsfähigkeit aus Frauensicht. Düsseldorf. NIEMANN, S. (1997): Lokale Agenda 21 - Neue Ära der Stadtplanung oder Dokument für die Schublade. In: Standort - Zeitschrift für angewandte Geographie, 21. Jg., H. 2/97, S. 31-36.

SELLE, K: (1994): Was ist bloß mit der Planung los? Erkundungen auf dem Weg zum kooperativen Handeln. Ein Werkbuch. Dortmunder Beiträge zur Raumplanung 69. Dortmund. SELLE, K. (Hrsg.) (1996): Planung und Kommunikation: Gestaltung von Planungsprozessen in Quartier, Stadt und Landschaft. Grundlagen, Methoden und Praxiserfahrungen. Wiesbaden. 


\section{Raumordnungspolitik der Europäischen Union - Bedeutung und Herausforderung für die Schweiz}

\section{Einleitung}

Die schweizerische Raumordnungspolitik verstand sich lange Zeit als binnenorientierte Aufgabe. Ihre Sicht war entsprechend introvertiert, und die Kontakte nach außen blieben relativ spärlich. Immerhin bemühen sich die Schweizer Grenzkantone seit längerem, räumliche Probleme entlang der Landesgrenzen gemeinsam mit ihren ausländischen Partnern pragmatisch zu lösen. In allen Grenzräumen der Schweiz haben sich Institutionen der grenzüberschreitenden regionalen Zusammenarbeit etabliert (z.B. Regio Basiliensis, Communauté de Travail du Jura, Regio Insubrica). Auf Bundesebene bestehen diesbezüglich jedoch erst wenige Ansätze. Zwar hat der Bundesrat im Bericht vom 22. Mai 1996 über die Grundzüge der Raumordnung Schweiz ${ }^{1}$ die räumliche Einbindung der Schweiz in Europa als eine Hauptstoßrichtung der schweizerischen Raumordnungspolitik bezeichnet. Die Konkretisierung und Umsetzung steht aber noch am Anfang.

Mehr und mehr tastet sich der Bund pragmatisch in einem Gebiet vor, das bisher in der schweizerischen EU-Integrationsdiskussion zu Unrecht eher geringe Beachtung gefunden hat. Die Möglichkeiten zur Einflußnahme auf europäische räumliche Entwicklungen, die durch aktives Mitwirken auf europäischer Ebene verbessert würden, werden wohl weitherum verkannt. Mit ihrer bislang situativen, vorab auf kantonaler Stufe verankerten Zusammenarbeit riskiert die Schweiz, ihre Landesinteressen nur ungenügend im internationalen Rahmen einbringen zu können und von bedeutenden Entwicklungen abgekoppelt zu werden. Zu nennen sind hier beispielsweise die internationale Verkehrsplanung (transeuropäische Netze, Hochgeschwindigkeitsbahnen) oder die immer mehr transnational, d.h. oberhalb der grenzüberschreitend-regionalen Ebene angesiedelten Raumordnungsinitiativen der EU.

Dieser Beitrag will aufzeigen, da $\beta$ in der schweizerischen und in der europäischen Raumordnungspolitik weitgehend deckungsgleiche Vorstellungen bestehen, daß die Schweiz ein großes Interesse an einer Aufwertung der europäischen Raumordnungspolitik hat und sie sich daher auf diesem Gebiet stärker engagieren sollte. ${ }^{2}$

\section{2. «Europa» \\ in der schweizerischen Raumordnungspolitik}

Ein Blick in den «Raumplanungsbericht $1987 »^{3}$ hinsichtlich Aussagen betreffend Europa oder grenzüberschreitender Zusammenarbeit macht deutlich, daß mit lediglich 2 von 160 Seiten (S. 86-87), die den internationalen Aspekten gewidmet waren, dieser Thematik noch vor 10 Jahren eine nur nachrangige Bedeutung zu$\mathrm{kam}$. In der Zwischenzeit hat die Bundes-Raumplanung zumindest auf konzeptionell-strategischer Ebene einen markanten Wandel vollzogen. In den Grundzügen der Raumordnung ist die Einbindung der Schweiz in die europäische Raumordnung zu einer von vier Hauptstoßrichtungen der schweizerischen Raumordnungspolitik geworden, neben «städtische Räume ordnen», «ländliche Räume stärken» und «Natur und Landschaft schonen» (vgl. Abb. 1).

Die Leitidee der «Grundzüge» ist das vernetzte Städtesystem Schweiz (vgl. Abb. 2). Dabei handelt es sich um eine modernisierte Variante der schon seit langem vorherrschenden Zielvorstellung der «dezentralen Konzentration». Durch eine hervorragende Vernetzung der Städte und der ländlichen Regionen sollen im Interesse der Wettbewerbsfähigkeit des Wirtschaftsstandortes Schweiz die wirtschaftlichen Potentiale der verschiedenen Teilräume vermehrt gebündelt und Synergien nutzbar gemacht werden. Das Land soll damit insgesamt gestärkt werden. Gleichzeitig soll die für das mehrsprachige und föderalistische Land wichtige dezentrale Siedlungsstruktur erhalten und der Landschaftsraum vor weiterer Zersiedlung bewahrt werden. Das vernetzte Städtesystem Schweiz macht nicht an der Landesgrenze halt, sondern fügt sich explizit in das europäische Städtenetz ein.

Die «Grundzüge» von 1996 haben also eine markante Aufwertung der Europathematik mit sich gebracht. Interessant ist auch ein inhaltlicher Vergleich von «Raumplanungsbericht» und «Grundzügen». Noch 1987 ging es in erster Linie um die regionale grenzüberschreitende Zusammenarbeit auf herkömmlichen Gebieten der Raumplanung (z. B. Abstimmung der Richtpläne mit dem grenznahen Ausland). Daneben kam auch der

Daniel Wachter, PD Dr., Stabsstelle für Raumökonomie, Bundesamt für Raumplanung, 3003 Bern 
CEMAT $^{4}$ im Rahmen des Europarates noch eine gewisse Bedeutung zu. 1996 sind diese Themen zwar nicht verschwunden, im Zentrum stehen jedoch funktionale und großräumige Fragen. Es geht vorab um die Einbindung der Schweiz in die europäischen Städte- und Infrastrukturnetze.

\section{Abb. 1 \\ Strategien der Raumordnung Schweiz}

\section{Städtische Räume}

ordnen

Vernetztes Städtesystem Schweiz

Erneuerung und Stärkung der Städte

Agglomerationen in ihrer Ausdehnung begrenzen und räumlich strukturieren

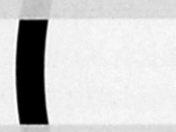

Eine Zukunft für de Lebens- und Wirtschaftsraum Schweiz

Vernetztes System von Städten und ländlichen Räumen

An Nachhaltigkeit orientierte räumliche Entwicklung

Siedlungsentwicklung nach innen

Natur- und

Landschaftsraum schonen

Landschaft von Überbauungen und neuen Belastungen freihalten

Landschaft großräumig und differenziert ordnen

Nutzungsvielfalt wiederherstellen

Natürliche Lebensgrund lagen und einheimische Pflanzen- und Tierwelt schützen
Die Schweiz in Europa einbinden

Den Blick gegen außen richten

Zusammenarbeit der Grenzregionen und -städte

Beitrag und Zusammenarbeit für eine europäische Raumordnung

Quelle: Bundesamt für Raumplanung (Herausgeber), 1996: Bericht des Bundesrates vom 22. Mai 1996 über die Grundzüge der Raumordnung Schweiz. EDMZ, Bern 1996. S. 37

Abb. 1 Strategien der Raumordnung Schweiz.

\section{Abb. 2 \\ Vernetztes Städtesystem Schweiz}

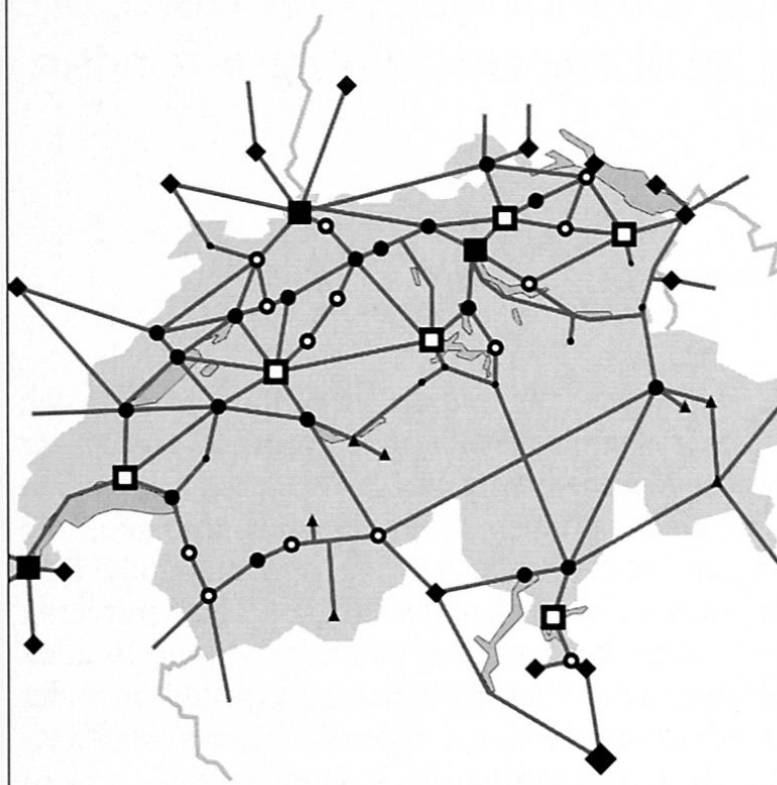

großstädtische Agglomerationen von internationaler Bedeutung

- Agglomerationen von nationaler Bedeutung

- mittelstädtische Agglomerationen von regionaler Bedeutung

- kleinstädtische Agglomerationen von regionaler Bedeutung

- Regionalzentren

- große Tourismuszentren

- ausländische Zentren

Datengrundlagen: Bundesamt für Statistik: Volkszăhlung 1990 sowie Hotel- und Parahotelleriestatistik. Bern

Quelle: Bundesamt für Raumplanung (Herausgeber), 1996: Bericht des Bundesrates vom 22. Mai 1996 über die Grundzüge der Raumordnung Schweiz. EDMZ, Bern 1996. S. 43

Abb. 2 Vernetztes Städtesystem Schweiz.

\section{Raumordnungspolitik der Europäischen Union ${ }^{5}$}

Konzeptionell und im Vollzug ist die schweizerische Raumordnungspolitik heute eine Gemeinschaftsaufgabe der eng koordinierten Politikbereiche Raumplanung und Regionalpolitik. Erstere ist mit ihren spezifischen Instrumenten eher im Bereich «Konzentration» tätig, letztere eher im Bereich der «Dezentralisation». Während die schweizerische Raumordnungspolitik heute also durch eine enge Kooperation und Koordination zwischen Regionalpolitik und Raumplanung gekennzeichnet ist, liegt auf der Seite der EU das Schwergewicht bei der Regionalpolitik und der regionalwirtschaftlich relevanten Infrastrukturpolitik. Die Re- 
gionalpolitik im engeren Sinne konnte sich schneller und weitgehend unabhängig von der Raumordnung bzw. Raumplanung entwickeln, weil sie über eigene finanzielle Mittel verfügte. Hierbei ist auf die drei Strukturfonds Europäischer Ausrichtungs- und Garantiefonds für die Landwirtschaft, Abteilung Ausrichtung (EAGFL-A), Europäischer Sozialfonds (ESF) und Europäischer Fonds für Regionale Entwicklung (EFRE) hinzuweisen ${ }^{6}$.

Über die Strukturfonds werden einerseits Maßnahmen auf Initiative der Mitgliedstaaten mitfinanziert, wobei die EU einen Rahmen bezüglich Zielvorgaben setzt. Es sind heute sechs Ziele bzw. Zielgebiete, welche künftig - gestützt auf die Agenda 2000, das Strategiedokument für die künftige EU-Politik im Zusammenhang mit der Osterweiterung - auf drei reduziert werden sollen.

Andererseits verfügt die EU mit den Gemeinschaftsinitiativen über einige weitere Ansatzpunkte für raumordnungspolitisches Handeln. Gemeinschaftsinitiativen werden auf der Grundlage von Leitlinien ausgearbeitet, die die Europäische Kommission selbst aufstellt. Sie sollen zur Lösung von Problemen beitragen, die für die Gemeinschaft von besonderem Interesse sind. Die Gemeinschaftsinitiativen werden ebenfalls aus den Strukturfonds finanziert. Im vorliegenden Zusammenhang ist insbesondere die INTERREG-Initiative ${ }^{7}$ relevant (vgl. dazu weiter unten).

Während die Regionalpolitik zunehmend vergemeinschaftet wurde, ist die Raumordnung/Raumplanung auf dem Gebiet der EU bis heute in nationaler Zuständigkeit verblieben. Diese Situation einer in nationaler Kompetenz verbliebenen Raumordnung und einer weitgehend vergemeinschafteten Regionalpolitik wurde von seiten der Wissenschaft und insbesondere von den für die Raumordnung zuständigen Ministern der Mitgliedstaaten schon lange als unbefriedigend empfunden. Wegen der intensiveren Integration zwischen den EUMitgliedstaaten auf inzwischen vielen raumwirksamen Politikfeldern und insbesondere wegen der starken Rolle der gemeinschaftlichen Regionalpolitik hätte sie in der EU schon längst stärker institutionalisiert werden müssen.

Die Mitgliedstaaten haben der EU bis zum heutigen Tage jedoch keine Kompetenzen im Bereich der Raumplanung erteilt. Folglich hat «Brüssel» keine raumplanerischen Befugnisse. Da verschiedene Bereiche der Gemeinschaftspolitik jedoch räumliche Auswirkungen haben, wird es immer dringlicher, die Beschlüsse der Europäischen Kommission auf der Grundlage eines integrierten, von der Raumplanung ausgehenden Ansatzes zu koordinieren.

Um diesem Bedürfnis gerecht zu werden, haben die Mitgliedstaaten der EU allerdings keine neuen Strukturen innerhalb der Gemeinschaftsorgane geschaffen. Seit 1989 finden vielmehr informelle Treffen der Raumplanungsminister statt. Zudem wurde ein Ausschuß für Raumentwicklung (ARE) geschaffen, der ausschließlich aus Raumplanungsbeamten der nationalen Raumplanungsbehörden besteht. Zuständig für die $\mathrm{Ge}-$ schäftsführung des ARE ist die Generaldirektion XVI «Regionalpolitik und Kohäsion».

Dieser informelle Ministerrat hat sich in den letzten Jahren über die laufende Abstimmung raumordnungspolitischer Fragen hinaus intensiv der Aufgabe gewidmet, ein europäisches Raumordnungskonzept zu entwerfen. Über verschiedene Phasen ist bis im Sommer 1998 ein «vollständig überarbeiteter Entwurf» für ein «Europäisches Raumentwicklungskonzept» (EUREK) erarbeitet worden. ${ }^{8}$ Es ist beabsichtigt, das EUREK in der ersten Jahreshälfte 1999 definitiv zu verabschieden. Mit dem EUREK werden keine zusätzlichen Kompetenzen für die Gemeinschaftspolitik geschaffen. Es ist auch rechtlich nicht bindend. Es ist dem auch in der Schweiz maßgeblichen raumordnungspolitischen Koordinationsgedanken verpflichtet. Es soll - ähnlich den Grundzügen der Raumordnung Schweiz - als Orientierungsrahmen für die bereits bestehenden Sachpolitiken und Programme dienen und damit zu einer besseren räumlichen Kohärenz der raumwirksamen Politiken der Europäischen Union beitragen. Das EUREK kann die Zusammenarbeit zwischen den Mitgliedstaaten sowie zwischen ihnen und der Europäischen Kommission verbessern.

Das EUREK verfolgt drei grundlegende Ziele: ${ }^{9}$

- Wirtschaftlicher und sozialer Zusammenhalt (Ausgleichsziel)

- Nachhaltige Entwicklung (Erhaltungsziel)

- Ausgeglichene Wettbewerbsfähigkeit im europäischen Raum (Entwicklungsziel)

und enthält drei Handlungsfelder: ${ }^{10}$

- Förderung eines ausgewogenen und polyzentrischen Städtesystems

- Schaffung gleichwertiger Zugangsmöglichkeiten zu Infrastruktur und Wissen

- Intelligente Nutzung und Entwicklung des natürlichen und kulturellen Erbes.

Diese Ziele und Handlungsfelder basieren auf einer Problemanalyse" reiche mit den aufgeführten weiteren Teilaspekten (Auswahl) unterschieden wurden:

- Veränderung der Städtestruktur (zunehmende Hierarchisierung und Polarisierung der Städtestruktur, anhaltende Flächenausdehnung der Städte und Zersiedlung des ländlichen Umlandes, zunehmende soziale Segregation in den Städten, mangelnde Verbesserung der städtischen Umweltqualität).

- Strukturwandel im ländlichen Raum (zunehmende Interdependenzen und Abhängigkeiten von städtischen Gebieten, weitere Ausdifferenzierung der Problemlagen der einzelnen ländlichen Regionen, ökonomische Bedeutung der Landwirtschaft zunehmend nur noch in agrarisch hoch produktiven Gebieten, Entwicklung der agrarisch weniger produktiven Gebiete je nach Potential zu Nischenanbietern im Nahrungsmittel-/Tourismussektoroder zu Entleerungsräumen). 
- Probleme im Bereich derVerkehrs-, Kommunikationsund Bildungsinfrastruktur (verbleibende Rückstände und Lücken entlang von Binnen- und Außengrenzen der Europäischen Union, zunehmende Überlastung und Engpässe, vor allem aufgrund steigenden Verkehrsaufkommens, unausgeglichene Erreichbarkeit in Europa, Konzentrationstendenzen entlang weniger Entwicklungskorridore, Disparitäten bei der Verbreitung von Innovationen und Wissen).

- Anhaltender Druck auf Europas Natur- und Kulturerbe (Verlust von Biodiversität und Naturräumen, Angebotsknappheit und Verschmutzung der Wasserressourcen, zunehmender Druck auf Kulturlandschaft, steigender Druck auf das europäische Kulturerbe).

\section{Das EUREK aus schweizerischer Sicht}

Zwischen der Problemsicht im EUREK und in den Grundzügen der Raumordnung Schweiz besteht über weite Strecken Deckungsgleichheit. Dasselbe trifft auch auf die Handlungsansätze und Strategien zu. ${ }^{12}$

Das erste Handlungsfeld des EUREK (Förderung eines ausgewogenen und polyzentrischen Städtesystems) ist bezüglich der konzeptionellen Inhalte zu trennen in Elemente, die das Städtesystem betreffen, und solche, die die Stadt-Land-Beziehungen im Auge haben. Die Aussagen in den Grundzügen der Raumordnung zum vernetzten Städtesystem Schweiz, zur Erneuerung und Stärkung der Städte und zur Begrenzung der Ausdehnung der Agglomerationen sind jenen des EUREK sehr ähnlich. Auch die EUREK-Elemente bezüglich Stärkung der ländlichen Räume und der Zusammenarbeit mit den städtischen Räumen weisen eine große Übereinstimmung mit der Strategie der «Grundzüge» auf.

Die Elemente des zweiten Handlungsfeldes des EUREK (Schaffung gleichwertiger Zugangsmöglichkeiten zu Infrastruktur und Wissen) finden sich fast vollständig in der schweizerischen Strategie «ländliche Räume stärken» wieder, spielt doch die Erschließungspolitik betreffend Verkehr und zunehmend Telekommunikation auch in der Schweiz ein große Rolle.

Das dritte EUREK-Handlungsfeld (intelligente Nutzung und Entwicklung des natürlichen und kulturellen Erbes) findet in der Strategie «Natur und Landschaft schonen» der schweizerischen "Grundzüge» sein $\mathrm{Ge}$ genstück, das ebenfalls über weite Strecken deckungsgleiche Inhalte aufweist.

Die schweizerische Raumordnungsstrategie «Schweiz in Europa einbinden» findet im EUREK kein explizites Pendant. Doch auch aus EU-Sicht ist und bleibt die Zusammenarbeit in Grenzregionen und transnational ein Vorrangthema, wie in der Agenda 2000 deutlich festgehalten wurde.

Aufgrund des hohen Grades an Übereinstimmung in der Zielsetzung muß die Schweiz an einer Umsetzung des EUREK sehr interessiert sein. In mancher Beziehung ist die schweizerische Politik bereits stärker EUREK-kompatibel ausgerichtet als jene der EU. Beispielsweise postuliert das EUREK, dem steigenden Verkehrsaufkommen u. a. mittels Anlastung der externen Umweltkosten des Verkehrs und Verlagerung von Straßenverkehr auf die Schiene entgegenzutreten. ${ }^{13}$ Solche Grundsätze zur umweltverträglichen Bewältigung der Verkehrsströme auch in der Schweiz nicht unumstritten - stehen im Widerspruch zur real existierenden, straßenlastigen EU-Verkehrspolitik. Die Schweiz, deren Güterverkehrspolitik die Umsetzung der EUREK-Grundsätze beabsichtigt ${ }^{14}$, hat deshalb ein eminentes Interesse an einer Aufwertung der europäischen Raumordnungspolitik, welche deutlicheren Einflu $\beta$ auf die Sachpolitiken der EU, insbesondere in bezug auf Umweltschutz, Nachhaltigkeit und Verkehrspolitik, nehmen könnte.

Aus Sicht der Schweiz ist überdies zu begrüßen - und dies wird insbesondere auch in der Geographie mit Interesse zur Kenntnis genommen werden - , daß mit dem EUREK in Europa eine Anstrengung unternommen wird, explizit die räumlich-territoriale Dimension in der Politik stärker zu verankern. Darunter wird insbesondere auch die Berücksichtigung von besonderen physischgeographischen Rahmenbedingungen, wie z.B. denjenigen in den Gebirgsräumen, verstanden. ${ }^{15}$ Die Mühen der Schweiz mit der europäischen Integration liegen teilweise ja auch darin begründet, daß weite Teile der Bevölkerung befürchten, der grenzenlose europäische Binnenmarkt erlaube zu wenig Rücksichtnahme auf die Kleinräumigkeit und enge Kammerung der Schweiz und die besonderen Anliegen als Alpenstaat, etwa bezüglich Berglandwirtschaft oder Gütertransit durch die ökologisch sensiblen Alpen.

Nur nebenbei bemerkt ist aus der Warte der schweizerischen Raumordnungspolitik zu hoffen, daß diese europäische Initiative zur Verstärkung der territorialen Dimension der Politik auch innerhalb der Schweiz, wo im Zeichen der Liberalisierung, Privatisierung und Deregulierung gegenwärtig ganzheitliche, transdisziplinäre Ansätze wieder vermehrt in Frage gestellt werden, zu neuen Impulsen für die Raumordnungspolitik führt.

\section{Chancen einer vermehrten Zusammenarbeit}

Zwischen der schweizerischen und der im Entstehen begriffenen europäischen Raumordnungspolitik im umfassenden Sinn besteht also beträchtliche Übereinstimmung. Bemerkenswert ist zudem, daß die Schweiz auf diesem Gebiet schon heute in der Regel ein willkommener Partner der EU ist. Dies belegt etwa das INTERREGProgramm, das ausdrücklich auch die EU-Außengrenzen einbezieht und an dessen Programmsparte INTERREG II A (grenzüberschreitend-regionale Zusammenarbeit) die Schweiz bereits teilnimmt. Auch der EUREKEntwurf ${ }^{16}$ offeriert Nichtmitgliedstaaten der EU Möglichkeiten zur Mitwirkung. 
Dieses Angebot bezieht sich einerseits auf die Weiterarbeit am EUREK bis zu dessen Fertigstellung, aber auch auf die Teilnahme an Gemeinschaftsinitiativen wie der neuen Programmsparte INTERREG IIC ${ }^{17}$. Dies ist die jüngste INTERREG-Programmsparte. Sie ist für die Jahre 1997 bis 1999 mit einem Budget von 413 Millionen ECU ausgestattet. INTERREG IIC unterscheidet sich von INTERREG IIA durch eine größerräumige Betrachtung - transnationale anstelle von grenzüberschreitend-regionaler Zusammenarbeit, d.h. Zusammenarbeit vorab auf nationaler Ebene mit überregionaler Optik - und durch Ziele, die explizit auf die Raumplanung ausgerichtet sind. In bezug auf die Sachbereiche stehen somit Fragen des Verkehrs, der Siedlungsentwicklung, der nachhaltigen Entwicklung u.ä. im Zentrum. Es geht darum, die Raumplanung in den beteiligten Staaten vermehrt aufeinander abzustimmen und Problembereiche von internationaler und überregionaler Dimension gemeinsam einer Lösung zuzuführen. INTERREG IIC - es ist bereits eine Weiterführung im Rahmen einer INTERREG-III-Initiative ab dem Jahr 2000 geplant - soll in der Anfangsphase ein wichtiges Instrument zur Umsetzung des EUREK darstellen. Die Ideen des EUREK sollen also zunächst über eine verstärkte Zusammenarbeit der nationalen Raumplanungsbehörden gefördert werden.

Die Schweiz, die als EU-Nichtmitglied alle Kosten selber übernehmen muß, stellte für INTERREG II A - also die grenzüberschreitend-regionale Zusammenarbeit einen Rahmenkredit von insgesamt 24 Millionen Franken bereit und ist damit in der Lage, hier aktiv teilzunehmen. Der schweizerische INTERREG-Beschluß war eine Reaktion auf die ablehnende Volksabstimmung zur Frage des EWR-Beitritts und soll nun über die kleine Außenpolitik die Integration schrittweise und pragmatisch vorantreiben ${ }^{18}$. Dieser Bundesbeschluß läuft allerdings 1999 aus. Will die Schweiz in Zukunft weiterhin bei INTERREG mitarbeiten, muß sie neue Mittel bereitstellen, die insbesondere auch eine Teilnahme an den überregionalen und transnationalen Aktivitäten ermöglichen. Gegenwärtig sind in den Bundesverwaltung Vorbereitungsarbeiten für eine Botschaft an die eidgenössischen Räte über einen INTERREG-Nachfolgebeschluß im Gang, denen im Laufe des Jahres 1999 im Parlament hoffentlich Erfolg beschieden sein wird.

Ein vermehrtes Engagement der Schweiz wäre nicht nur ein Akt der Solidarität und ein konstruktiver Beitrag zur Gestaltung des zukünftigen Europa, sondern würde unserem Land auch erlauben, Einfluß auf die raumwirksamen Sektoralpolitiken der EU zu nehmen.

\footnotetext{
Anmerkungen

1 BBI 1996 III 556.

${ }^{2}$ Vgl. DANIEL WACHTER: Raumordnungspolitik. In: THOMAS COTTIER, ALWIN KOPSE (Hrsg.): Der Beitritt der Schweiz zur Europäischen Union: Brennpunkte und Auswirkungen. Schultheß Polygraphischer Verlag, Zürich 1998, 1203-1228.
}

${ }^{3}$ Bericht vom 14. Dezember 1987 über den Stand und die Entwicklung der Bodennutzung in der Schweiz (BBI 1988 I 871).

${ }^{4}$ CEMAT = Conférence Européenne des Ministres responsables de l'Aménagement du Territoire.

${ }^{5}$ DIETER BIEHL: Perspektiven der europäischen Raumentwicklungspolitik: Eine deutsche Sicht. Vortrag gehalten an der Tagung «Europäische Raumentwicklung - Herausforderungen für die schweizerische Raumplanung", Zürich, 21. November 1997 (unveröffentlichtes Manuskript); PHILIPPE DOUCET: Raumplanung in der Europäischen Union: Hin zu einer gemeinsamen, gegenüber Drittstaaten offenen Strategie. In: Informationshefte Raumplanung, Themenheft 4/97 zum Thema "Europa», Bern 1997, 18-20; HELMUT KARL, WILHELM HENRICHSMEYER (Hrsg.): Europäische Raumentwicklungspolitik: Notwendigkeit einer vertraglichen Verankerung? Bonner Schriften zur Integration Europas, Band 8, Bonn 1996; MICHAEL KRAUTZBERGER, WELF SELKE: Perspektiven der bundesstaatlichen Raumplanungspolitik in der Europäischen Union - Das Beispiel der Bundesrepublik Deutschland. Schriftenreihe für Städtebau und Raumplanung, Band 5, Wien 1996.

${ }^{6}$ EUROPÄISCHE KOMMISSION, GENERALDIREKTION XVI (Regionalpolitik und Kohäsion): Europa fördert die regionale Entwicklung, Luxemburg 1996.

7 INTEGRATIONSBÜRO EDA / EVD: INTERREG Grenzüberschreitende Zusammenarbeit zwischen der Schweiz und der Europäischen Union, Bern 1996; ALAIN SCHALLER: INTERREG IIA - L'initiative de promotion de la coopération régionale transfrontalière. In: Informationshefte Raumplanung. Themenheft 4/97 zum Thema "Europa", Bern 1997. 33-34; ALAIN SCHALLER: INTERREG ॥A - Grenzüberschreitende Zusammenarbeit. In: Die Volkswirtschaft, Heft 12/1997, 27-30.

${ }^{8}$ EUREK / Europäisches Raumentwicklungskonzept. Vollständig überarbeiteter Entwurf, Noordwijk. 8. Juni 1998.

9 EUREK, deutsche Fassung vom 8. Juni 1998, 1.

10 EUREK, deutsche Fassung vom 8. Juni 1998, 50.

11 EUREK, deutsche Fassung vom 8. Juni 1998, $21 \mathrm{ff}$.

12 PETER GÜLLER, ROBERTO DE TOMMASI: Grundlagen der räumlichen Vernetzung mit Europa. Forschungsbericht zuhanden des Bundesamtes für Raumplanung, Bern 1996; PETER GÜLLER: Raumordnungspolitik Schweiz und Europa - Thesen zur Ausgestaltung der Beziehungen zwischen der schweizerischen und europäischen Regional- und Raumordnungspolitik zuhanden der Raumordnungskonferenz des Bundes, Bern 1997 (unveröffentlichtes Manuskript).

${ }^{13}$ EUREK, deutsche Fassung vom 8. Juni 1997, 62.

14 Bei der Niederschrift dieses Beitrages im Sommer 1998 ist noch offen, ob sich die umweltorientierte Verkehrspolitik der Schweizer Regierung in die Tat umsetzen läßt (vgl. zum Beispiel die Volksabstimmungen über die leistungsabhängige Schwerverkehrsabgabe, Finanzierung der Neuen EisenbahnAlpentransversalen NEAT). Der Autor geht jedoch von der Hypothese aus, daß die Schweizer Regierung beim Volk letztlich Unterstützung findet.

15 EUREK, deutsche Fassung vom 8. Juni 1997, 10 und 86. 16 EUREK, deutsche Fassung vom 8. Juni 1998, 93.

17 ARMAND MONNEY: Transnationale Zusammenarbeit in der Raumplanung: Herausforderung und Chance für die Schweiz. In: Informationshefte Raumplanung, Themenheft 4/97 zum Thema "Europa", Bern 1997, 13-15; WELF SELKE: INTERREG IIC: Experimentierfeld transnationaler Zusammenarbeit. In: Informationshefte Raumplanung, Themenheft 4/97 zum Thema «Europa", Bern 1997, 16-17.

${ }_{18}$ Bericht vom 7. März 1994 über die grenzüberschreitende Zusammenarbeit und die Mitwirkung der Kantone an der Außenpolitik (BBI 1994 II 620). 